\title{
Helicobacter Pylori- Factors of Virulence and Persistence: The Ability to Colonise the Human Stomach for Extended Periods Despite Host Immune and Inflammatory Responses.
}

\author{
Aus Molan \\ Research and in-vitro Diagnostics/Abacus ALS/Australia
}

\begin{abstract}
Helicobacter pylori is perhaps the most prevalent and successful human pathogen worldwide. Since it was initially suggested in 1983 by Marshall and Warren to be implicated in gastritis and peptic ulcer disease, $H$. pylori has also been implicated in gastric carcinoma and was classified as a class I carcinogen. Over half of the world's population is colonised with this gram-negative bacterium with most infections being acquired early in life and may persist for the life of the individual. H. pylori infection represents a key factor in the etiology of various gastrointestinal diseases, ranging from chronic active gastritis without clinical symptoms to peptic ulceration, gastric adenocarcinoma, and gastric mucosa-associated lymphoid tissue lymphoma. While infected individuals mount an inflammatory response that becomes chronic, along with a detectable adaptive immune response, these responses are ineffective in clearing the infection. H. pylori has unique features that allow it to reside within the harsh conditions of the gastric environment, and also to evade the host immune response. Therefore, disease outcome is the result of the complex interplay between the host and the bacterium. Host immune gene polymorphisms and gastric acid secretion largely determine the bacterium's ability to colonise a specific gastric niche. Bacterial virulence factors such as the cytotoxin-associated gene pathogenicity island-encoded protein CagA and the vacuolating cytotoxin VacA aid in this colonisation of the gastric mucosa and subsequently seem to modulate the host's immune system. In this review, the various virulence factors expressed by this bacterium and how they interact with the host epithelium to influence pathogenesis are discussed.
\end{abstract}

KEYWORDS : Helicobacter pylori, cytotoxin-associated gene A, CagA, vacuolating cytotoxin A, VacA, bacterial virulence, host immunity, gastric carcinoma.

\section{INTRODUCTION}

Over three decades ago, Robin Warren and Barry Marshall definitively identified Helicobacter pylori by culturing an organism from gastric biopsy specimens that had been visualised for almost a century by pathologists [1]. In 1994, H. pylori was recognised as a type I carcinogen, and now it is considered the most common etiologic agent of infection-related cancers, which represent $5.5 \%$ of the global cancer burden [2]. This discovery resulted in the awarding of the 2005 Nobel Prize in Physiology or Medicine to Robin Warren and Barry Marshall for their "discovery of the bacterium Helicobacter pylori and its role in gastritis and peptic ulcer disease." These organisms are helical shaped, Gram-negative bacteria that selectively colonise the gastric epithelium. The bacterium is urease, catalase, and oxidase positive, and possesses three to five polar flagella that are used for motility. In addition, the majority of $H$. pylori strains express virulence factors that have evolved to affect host cell signaling pathways. One of the many unique characteristics of $H$. pylori is the capacity to persist for decades in the harsh gastric environment due to an inability of the host to eliminate the infection. Unlike other viruses and bacteria, $H$. pylori has evolved the ability to colonise the highly acidic environment found within the stomach by metabolising urea to ammonia via urease, which generates a neutral environment enveloping the bacterium. At least half the world's population is infected with Helicobacter pylori, and the majority of colonised individuals develop a co-existing chronic inflammation. In the majority of hosts, $H$. pylori colonisation does not cause any symptoms [3]. However, long-term carriage of $H$. pylori considerably increases the risk of developing gastric diseases. Among infected individuals, approximately $10 \%$ develop peptic ulcer disease, 1 to $3 \%$ develop gastric adenocarcinoma, and $<0.1 \%$ develop mucosa-associated lymphoid tissue (MALT) lymphoma [4]. The twin hallmarks of the interaction between Helicobacter pylori and humans are its persistence during the life of the host, and the host's responses to its continuing presence. This conflict appears paradoxical, but both the microbe and the host adapt to the other in the form of a long-standing dynamic equilibrium. Our understanding of the phenomena underlying these interactions is growing. These relationships are important, because of the major role of $\mathrm{H}$. pylori in promoting the risk of peptic ulcer disease [5] and noncardia adenocarcinoma of the stomach, and because of the emerging evidence that gastric $\mathrm{H}$. pylori colonisation has a protective role in relation to severe gastro-oesophageal reflux disease and its sequelae, 
Barrett oesophagus and adenocarcinoma of the oesophagus [3]. New studies suggest other important impacts of $H$. pylori colonisation on human physiology [6].The number of peer-reviewed publications on Helicobacter has rapidly increased, from less than 200 in 1990 to approximately 1,500 per year over the last few years (PubMed [www.pubmed.gov]). Although the prevalence of $H$. pylori in the Western world is decreasing, gastric colonisation by $H$. pylori remains widespread in the developing world. This review focuses on the various virulence factors expressed by this bacterium. Also discussed is how these factors interact with the host epithelium to influence pathogenesis.

\section{VIRULENCE, PERSISTENCE, AND HOST DEFENSE RESPONSES}

The remarkable diversity of $H$. pylori may be viewed as evidence of a versatile population that is able to maximise resource utilisation in a variety of niches and micro-niches and to avoid host constraints. The generation of diversity typically involves endogenous (point) mutations and recombination; H. pylori has mechanisms for each. Most strains of H.pylori would be considered to have this hyper-mutator phenotype which favours the emergence of variants after selective pressure [7]. A good example of efficient adaptive point mutation in the H. pylori bacterial population is the rapid development of high-level resistance to commonly used antibiotics, such as clarithromycin.

H. pylori cells are also highly competent for uptake of DNA from other Helicobacter strains. Analysis of sequences shows strong evidence of recombination between strains, to the degree that clonal lineages are largely obscured [8]. It has been shown that substantial intra-genomic recombination occurs, based largely on the presence of repetitive DNA sequences [8]. These DNA repetitions allow for high-frequency deletion and duplication, including slipped-strand mispairing. However, because H. pylori is naturally competent, any genetic element that is lost may be regained from either unaffected sectors of the population of that strain or from another strain. A lack of mismatch-repair systems may increase the frequency of random variation, but it also may facilitate gene conversion, which minimises genomic diversity of those alleles present in multiple copies. Thus, H. pylori can maximise a diversity of sequences under strong selective pressure, while maintaining alleles that are critical for its lifestyle.

\section{HUMAN INTERACTION DOMAIN 1- CAGA}

In 1989, a strain-specific $H$. pylori gene, cagA, was identified [9], which now has been recognised as a marker for strains that confer increased risk for peptic ulcer disease and gastric cancer [10]. No homologs are known for $\operatorname{cagA}$ in other Helicobacter species or in other bacteria, suggesting that it reflects a human gastricspecific gene. cagA is a marker for the $35-$ to $40-\mathrm{kb}$ cag (cytotoxin-associated gene) pathogenic island. The island contains genes encoding a type IV secretion system, which in other bacteria inject macromolecules (i.e., DNA and proteins, such as pertussis toxin) into host cells. Segal et al [11] concluded that one substrate for the type IV system in $H$. pylori is the cagA product, which is injected into epithelial cells, both in vitro and in vivo. In many strains, the cagA protein contains tyrosine-phosphorylation sites that are recognised by the host cell Src kinase. Once phosphorylated, cagA interacts with SHP-2, a tyrosine phosphatase, which affects spreading, migration, and adhesion of epithelial cells. This phenomenon can be assessed in vitro by a change in epithelial cell morphology to the scattered, or "hummingbird," phenotype.

\section{HUMAN INTERACTION DOMAIN 2- VACA}

Culture supernatants from some $H$. pylori strains release a high-molecular weight multimeric poreforming protein refered to as vacuolating cytotoxin $\mathrm{A}(v a c A)$, that causes massive vacuolation in epithelial cell lines. As with $\operatorname{cag} A$, no close homologs of $v a c A$ exist in other Helicobacter species or in other bacteria, which suggests its importance in the specific relationship of $H$. pylori with the human stomach. However, unlike $c a g A$, $v a c A$ is conserved among all $H$. pylori strains, although significant polymorphism exists. VacA has several specific properties that may contribute to H. pylori persistence in the gastric niche. (i) Firstly, it forms pores in epithelial cell membranes, allowing egress of anions and urea. This is important since urea hydrolysis, catalysed by $H$. pylori urease, protects against gastric acidity. (ii) VacA also induces loosening of epithelial tight junctions, potentially allowing nutrients to cross the mucosal barrier to $H$. pylori's gastric luminal niche. (iii) Recent in vitro work suggests that vacA may help H. pylori persistence by specific immune suppression. VacA blocks phagosome maturation in macrophages, selectively inhibits antigen presentation in $\mathrm{T}$ cells, and blocks $\mathrm{T}$ cell proliferation.(iv) Besides these actions that may benefit $H$. pylori persistence, vacA also has direct celldamaging effects in vitro, inducing cytoskeletal changes, apoptosis, and suppression of epithelial cell proliferation and migration, as well as cell vacuolation. 


\section{IMMUNE EVASION AND MANIPULATION}

If a microbe is to persist in a vertebrate host, its biggest challenge is to avoid clearance by the immune system. Transient $H$. pylori colonisation has been documented in both primates and humans, implying that persistence does not inevitably follow acquisition. The race between $H$. pylori adaptation to a specific host and the development of effective immunity also implies the feasibility of vaccine development. However, following H. pylori acquisition, there is rapid host recognition in the forms of both innate and acquired immune responses, including generation of specific local and systemic antibodies [12]. Nevertheless, the lifelong colonisation by the bacterium despite the ubiquity and duration of the host recognition demonstrates the effectiveness of $H$. pylori's strategies to evade host immunity.

The first important step in bacterial host colonisation is to survive without tissue invasion- and the bulk of $\mathrm{H}$. pylori cells, if not all of them, reside in the gastric lumen beyond the reach of most host immune recognition and effector mechanisms. However, Peek et al. [12] also found that even in this niche, some H. pylori cells establish intimate contact with the surface epithelium. Some H. pylori proteins cross the epithelial barrier, and both innate and acquired immune systems are activated. Although not able to completely avoid immune activation, $\mathrm{H}$. pylori has evolved mechanisms to reduce recognition by immune sensors by downregulating the activation of immune cells. Innate immune system recognition of microorganisms involves TollLike Receptors (TLRs) that discriminate pathogen-associated molecular patterns. TLR stimulation triggers proinflammatory signalling through NF- $\mathrm{\kappa B}$ activation, and $\mathrm{H}$. pylori has evolved to minimise such stimulation. TLR5 recognises bacterial flagella such as those of Salmonella typhimurium but is not stimulated by H. pylori flagella. In addition, TLR9 recognises the largely un-methylated DNA of most bacteria, but the highly methylated H. pylori DNA is likely to go unrecognised. Moreover, due of lipid A core modifications, H. pylori LPS is anergic compared with that of other enteric bacteria, and while it stimulates a macrophage TLR4 response, it does not stimulate gastric epithelial TLR4. Although H. pylori is relatively camouflaged from innate immune sensors on cell surfaces, $\mathrm{cag}^{+}$strains do stimulate NF-kB activation in epithelial cells, apparently through recognition by Nod1 (an innate intracellular pathogen-recognition molecule that detects soluble components of bacterial peptidoglycan). How such components are delivered to the epithelial cytoplasm by the cag-encoded type IV secretion system remains unclear, but the resultant NF-kB-induced pro-inflammatory cytokine expression is an important and continuing stimulus to inflammatory cell infiltration and thus to pathogenesis.

H. pylori also activates the acquired immune system, as indicated by both the humoral and cellular recognition of its antigens although it has evolved to substantially down-regulate and avoid acquired immune effectors. Recognition by the acquired immune system requires antigen presentation, and H. pylori interferes with both the uptake and processing of antigens, partially through a vacA effect [13]. H. pylori also suppresses T cell proliferation, activation, and induces selective $\mathrm{T}$ cell apoptosis, again partially through specific vacA effects on signaling. It also evades host adaptive responses by mimicry of the gastric epithelial fucosylated (Lewis) antigens, and by antigenic variation of surface proteins. As this high-frequency antigenic variation occurs through mutation (usually slipped-strand mispairing) and intra-genomic recombination between homologous sequences, these genetic mechanisms are important contributors to immune evasion. Finally, H. pylori can also suppress less specific immune mechanisms such as phagocytosis [13]. The relative contributions of the different host manipulation and evasion strategies to $\mathrm{H}$. pylori persistence are not established, possibly differing in individual hosts, but the existence of these varied mechanisms implies that immune surveillance of the gastric lumen is powerful, and that bacterial survival requires its subversion

\section{CONCLUSIONS}

The role of H. pylori in gastroduodenal disease has become firmly established. H.pylori is an effective and significant bacterial pathogen. For example, motility allows H.pylori to escape the acidity of the stomach and burrow through and colonise the gastric mucosa in close association with the epithelium. Urease enzyme likely plays a significant role in the survival and growth of H.pylori in the stomach by creating an alkaline microenvironment. Other virulence determinants include adhesins for colonisation of mucosal surfaces, mediators of inflammation, and a vacuoloating cytotoxin that causes damage to host cells. Two decades of intense research into $\mathrm{H}$. pylori virulence factors such as the VacA and CagA proteins have revealed many aspects of the relationships between this bacterium, the gastric mucosal surface, and the induction of disease. Disease outcome is the result of the intricate, ongoing interplay between environmental, bacterial, and host factors. Strain-to-strain genetic variability in bacterial virulence factors such as $v a c A$ and $c a g A$ not only affects the ability of the organism to colonise and cause disease but also affects inflammation and gastric acid output. In the continuous interactions with the host, the bacteria are able to adapt by mutations and DNA rearrangements, rendering novel genotypes. On the host side, 
variations in the host immune response to the chronic presence of $H$. pylori directly impact H. pyloriassociated gastric disease and affect gastric acid output and thereby the density and location of $H$. pylori cells. Many of these $H$. pylori-host interactions have similarities with the interactions between the gut flora and the gastrointestinal tract and may serve as paradigms for the interactions between bacteria and their hosts. Although H.pylori is non-invasive, untreated infections last for life and persist despite significant host immune inflammatory responses.

\section{REFERENCES}

[1] J Warren, B Marshal. Unidentified curved bacilli on gastric epithelium in active chronic gastritis, Lancet, 1983, $1273-1275$.

[2] D Parkin, Bray F, Ferlay J, Pisani P. Global cancer statistics, 2002, CA Cancer J. Clin., 2005, 55, 74-108.

[3] R Peek, Blaser M. Helicobacter pylori and gastrointestinal tract adenocarcinomas, Nat. Rev. Cancer, $2002,2,28-37$.

[4] R Peek, Crabtree J. Helicobacter infection and gastric neoplasia, J. Pathol., 2006, 208, 233-248.

[5] A Nomura, et al. Helicobacter pylori cagA seropositivity and gastric carcinoma risk in a Japanese American population, J. Infect. Dis., 186, 2002, 1138-1144.

[6] T Azuma, et al. Gastric leptin and Helicobacter pylori infection, Gut, 49, 2001, 324-329

[7] B Bjorkholm, et al. Mutation frequency and biological cost of antibiotic resistance in Helicobacter pylori, Proc. Natl. Acad. Sci. U. S. A, 98, 2001, 14607-14612.

[8] S Suerbaum, et al. Free recombination with Helicobacter pylori. Proc. Natl. Acad. Sci. U. S. A., 95, 1998, $12619-12624$.

[9] T Cover, C Dooley, M Blaser. Characterization and human serologic response to proteins in Helicobacter pylori broth culture supernatants with vacuolizing cytotoxin activity, Infect. Immun., 58, 1990, 603-610.

[10] J Crabtree, et al. Mucosal IgA recognition of Helicobacter pylori $120 \mathrm{kDa}$ protein, peptic ulceration, and gastric pathology, Lancet; 338, 1991, 332-335.

[11] E Segal, J Cha, J Lo, S Falkow, L Tompkins. Altered states: involvement of phosphorylated CagA in the induction of host cellular growth changes by Helicobacter pylori, Proc. Natl. Acad. Sci. U. S. A., 96, 1999, 14559-14564.

[12] R Peek, et al. Heightened inflammatory response and cytokine expression to cagA Helicobacter pylori strains, Lab. Invest., 73, 1995, 760-770.

[13] M Molinari, et al. Selective inhibition of Ii-dependent antigen presentation by Helicobacter pylori toxin VacA, J. Exp. Med., 187, $1998,135-140$. 